\title{
A RARE CASE OF INTESTINAL OBSTRUCTION IN LATE PREGNANCY
}

\section{Shaival Bhatt' ${ }^{1}$ Manisha Laddad2, Nitin Kshirsagar³, Rajkumar Patange ${ }^{4}$}

1 Junior Resident, Department of Obstetrics and Gynaecology, Krishna Institute of Medical Sciences, Karad. ${ }^{2}$ Associate Professor, Department of Obstetrics and Gynaecology, Krishna Institute of Medical Sciences, Karad. 3 Professor, Department of Obstetrics and Gynaecology, Krishna Institute of Medical Sciences, Karad. 4 Professor, Department of Obstetrics and Gynaecology, Krishna Institute of Medical Sciences, Karad.

\section{ABSTRACT}

Intestinal obstruction complicating an otherwise normal pregnancy is very rare. Such cases are extremely difficult to diagnose, especially during late pregnancy. Patient usually presents with an acute abdomen. We present here a case of intestinal obstruction in late pregnancy and its outcome. High clinical suspicion is required with active intervention to increase favourable outcome in cases like these.

\section{KEYWORDS}

Intestinal Obstruction, Pregnancy, Acute Abdomen.

HOW TO CITE THIS ARTICLE: Bhatt S, Laddad M, Kshirsagar N, et al. A rare case of intestinal obstruction in late pregnancy. J. Evolution Med. Dent. Sci. 2016;5(63):4502-4503, DOI: 10.14260/jemds/2016/1027

\section{INTRODUCTION/CASE}

This patient, 23 year old, primigravida with 35-week pregnancy, presented with acute pain in abdomen since 1 day. It was not associated with any other complaints. On examination, her uterus was irritable. There were no other positive findings.

Patient had been admitted one week back for the similar complaints. On examination, she had mild uterine contractions and tocolytics were given.

One day later, patient had complaints of 4-5 episodes of vomiting. X-ray of erect abdomen and ultrasonography of abdomen was done. Both showed signs of small bowel obstruction. Blood work was within normal limits.

\section{Investigations}

- USG abdomen-pelvis showed signs of bowel obstruction.

- X-ray showed 9 air fluid levels suggesting small bowel obstruction.

Patient was managed conservatively. Ryles tube was passed with aspiration every two hourly. Patient was started on antibiotics and kept nil by mouth.

She delivered a healthy female child next day without complications. Initial symptoms of obstruction were relieved by conservative management.

Repeat ultrasonography of abdomen showed mild subacute obstruction with inflammatory strictures (? enteritis ? tubercular).

Patient had no complaints and was discharged with instructions for strict followup.

Financial or Other, Competing Interest: None.

Submission 30-06-2016, Peer Review 24-07-2016,

Acceptance 30-07-2016, Published 08-08-2016.

Corresponding Author:

Dr. Shaival Bhatt,

Dr. M. M. Laddad.

75/A Block, Malkapur,

Karad-415110.

E-mail: bhatt.shaival@gmail.com

DOI: $10.14260 /$ jemds/2016/1027

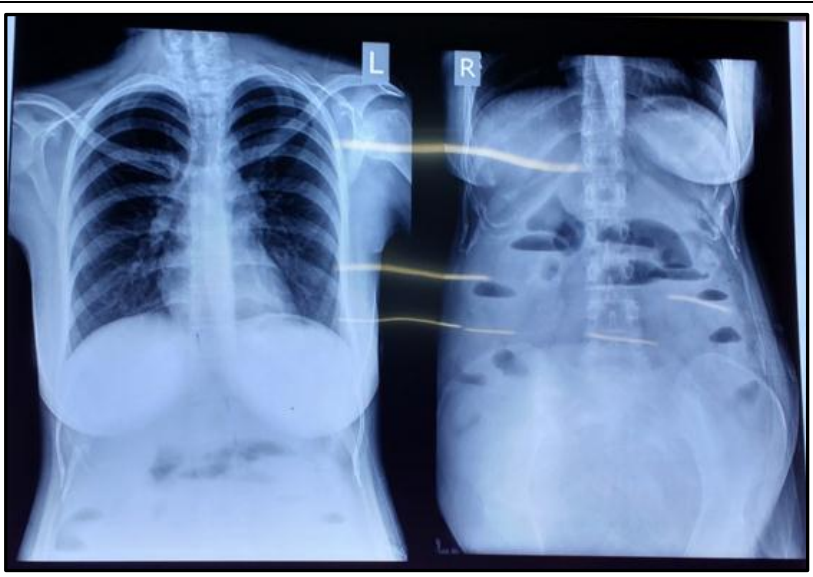

X-Ray Showing Air Fluid Levels

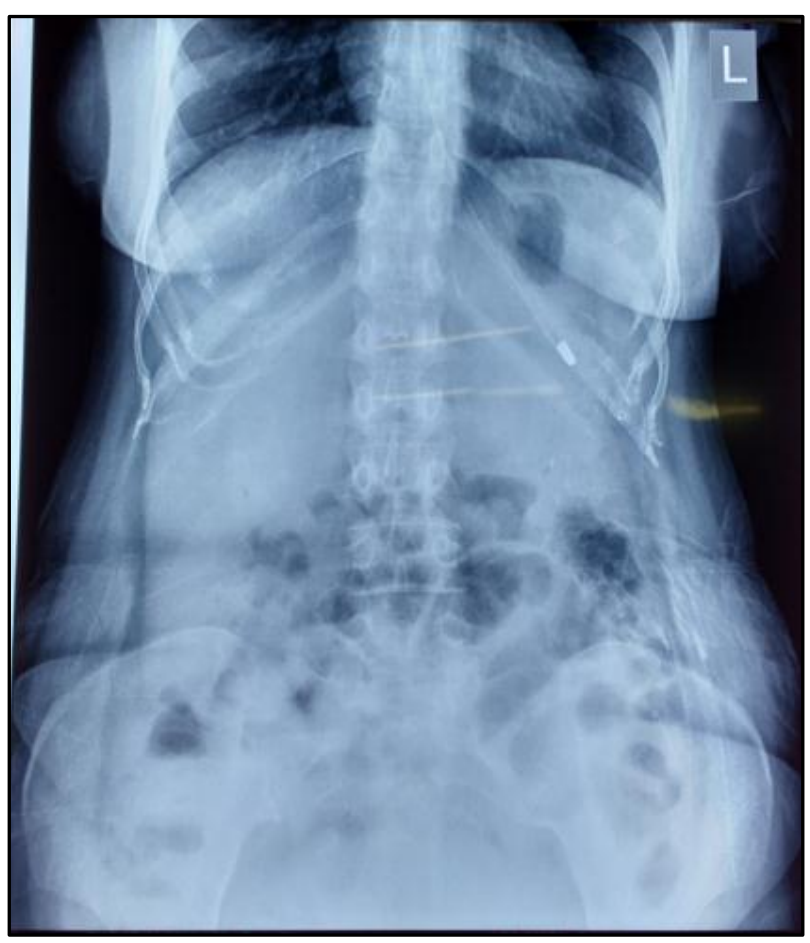

X-Ray Taken After Insertion of Ryles Tube 


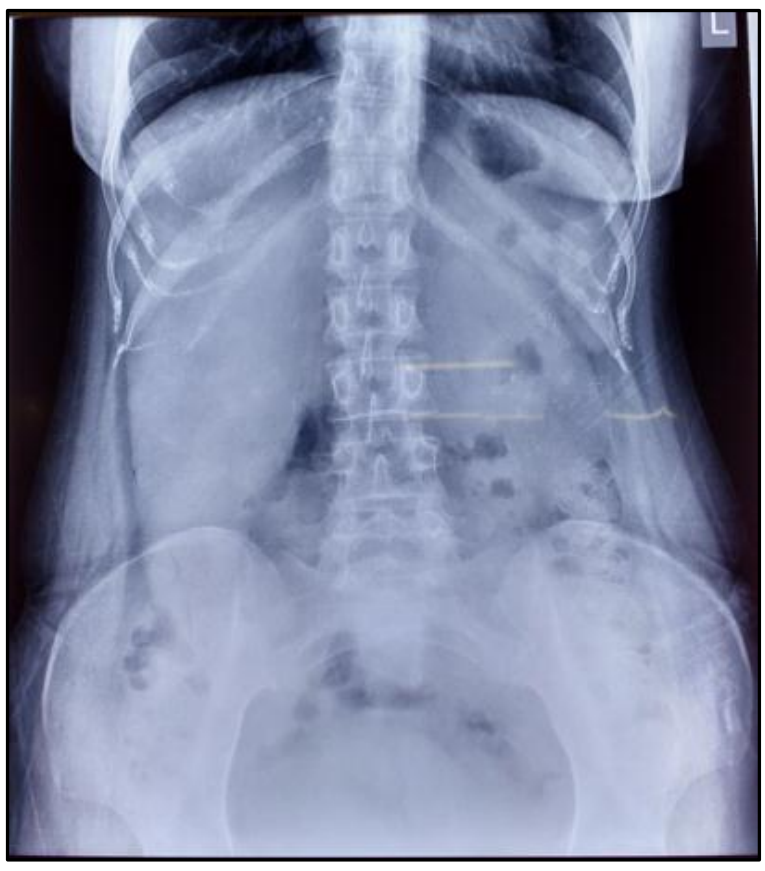

X-Ray After Patient Delivered.

\section{DISCUSSION}

Intestinal obstruction is relatively rare in pregnancy, but is its incidence is high in nonpregnant patients. The incidence is $1 \mathrm{in}$ 3000 pregnancies. According to research done in NorthWestern University of Chicago, "incidence is extremely rare in early pregnancy, but begins to increase between the fourth and fifth month as uterus becomes an abdominal organ. The peak incidence of bowel obstruction occurs in the eighth month when the foetal head descends into the pelvis, but it may also occur during delivery or the puerperium due to sudden change of size of uterus, which leads to anatomical changes in the relationship of abdominal organs. There is no relation between maternal age or parity and the risk of intestinal obstruction."(1)

According to their research, the causes of intestinal obstruction are same as in nonpregnant population, in that, previous abdominal surgery with resultant adhesions is the cause in approximately $60 \%$ of cases whereas the remaining aetiologies include volvulus, Crohn's disease, malignancy, and intussusceptions. Incarceration of hernias is relatively rare in pregnancy because the uterus displaces bowl loop from the inguinal and femoral rings as it enlarges.(1)

Even though causes of intestinal obstruction are similar in pregnant and nonpregnant, pregnancy predisposes these conditions. This is explained partly by the rearrangement of the abdominal organs in late pregnancy and partly by the physiological atony, which occurs due to endocrinal changes during pregnancy. According to study conducted by James D. W., from the obstetric point of view, there are four possible methods of treatment, depending upon whether the obstruction is acute or subacute. They are as follows:

1. Immediate laparotomy. Lower-segment caesarean section followed by treatment of the obstruction on general surgical principles.

2. Immediate laparotomy. Relief of the obstruction without disturbing the pregnancy. Morphine and progesterone to postpone labour as long as possible.

3. Surgical induction of labour. Spontaneous relief of the obstruction after delivery.
4. Surgical induction of labour. Laparotomy immediately after delivery if the obstruction is unrelieved.

The third and fourth are usually used for cases of subacute intestinal obstruction.(2)

According to findings of Shakina et al, adhesions are the commonest cause of intestinal obstruction in pregnancy. The incidence and complication rates increase with gestational age particularly in the third trimester. The risk of gestational intestinal obstruction increases as the uterus enlarges. Other causes include volvulus (23\%), intussusceptions (5\%), hernia $(3 \%)$, carcinoma $(1 \%)$, appendicitis $(1 \%)$, and idiopathic "ileus" (8\%).(3)

As stated by Beck et al in their research, the initial treatment consists of intestinal obstruction starts with nasogastric aspiration and aggressive IV fluids to correct electrolyte disturbances. CT with contrast is the gold standard investigation. Failure of conservative treatment and demonstration of complete obstruction on CT are indications for early surgery as persistence will contribute to an increase in mortality and morbidity. Perinatal death from hypoxia secondary to maternal hypovolaemia, sepsis, and peritonitis has been reported.(4) Maternal nutritional deficiencies can occur if the patient is kept nil per os (NPO) for a prolonged period.

If foetal distress is present or if there is inadequate exposure at laparotomy, delivery by caesarean section should precede the relief of obstruction.(4)

\section{CONCLUSION}

To conclude, it should be kept in mind that intestinal obstruction is a very rare occurrence and hence possess great challenge in diagnosing. A high index of suspicion is needed clinically to diagnose such a condition. Many of the signs and symptoms of labour and late pregnancy overlap with those of intestinal obstruction, which makes arriving at the accurate diagnosis even more difficult. Hence, with adequate suspicion of the condition and prompt timely intervention, chances of favourable outcome can be increased.

Fortunately in this case, patient could be managed conservatively with favourable outcome of mother and the foetus.

\section{ACKNOWLEDGMENT}

I would like to thank my associate professor for guiding me through this case report. I would also like to thank my head of unit and head of the department for giving me this opportunity. I am grateful to my colleagues for their support.

\section{REFERENCES}

1. Pandolfino J, Vanagunas A. Gastrointestinal complications of pregnancy. Gastroenterology Part 4, 2000;6:1-2.

2. James DW. Intestinal obstruction during late pregnancy. British Medical Journal 1946;1(4447):485-6.

3. Rauff S, Chang SKY, Tan EK. Intestinal obstruction in pregnancy: a case report. Case Reports in Obstetrics and Gynaecology Article ID 564838, 2013;2013:2 pages.

4. William BW. Intestinal obstruction in pregnancy. Obstetrics and Gynaecology 1974;43(3):374-8. 